\title{
On double framed $B(T)$ soft Fuzzy ideals and doubt double framed soft Fuzzy algebras of BF-algebras
}

\author{
COMSATS University Islamabad, Pakistan \\ Received: 13 May 2018, Accepted: 31 January 2019 \\ Published online: 12 May 2019.
}

Muhammad Bilal Khana, Gul Sanab and Muhammad Iftikharc

\begin{abstract}
In this article, we aim to consider a new kind of double framed B(T)-soft fuzzy ideal (briefly, DFB(T)SF-deal), doubt double framed T-soft fuzzy-algebra (briefly, DDF-soft fuzzy-algebra) and doubt double framed T-soft fuzzy ideal (briefly, DDF-soft fuzzy-ideal) of BF-algebra over a $(U,[0,1])$. The notions of DF soft fuzzy-deals, DDF-soft fuzzy-algebras and DDF-soft fuzzy-ideals are defined and their basic properties are investigated. With the support of examples, these notions are illustrated. Further, in DF-soft fuzzy-ideal the union product are studied and proved that their union product is also a DF-soft fuzzy-deal. Similarly, this property is also illustrated in DDF-soft fuzzy-algebra and DDF-soft fuzzy-ideal by using intersection-product. Furthermore, in this article the results which are proved for DDF-soft fuzzy-algebra of BF-algebra also satisfied for DDF-soft fuzzy-algebra of BCI/BCI-algebra except the concept of ideals of BF-algebra.
\end{abstract}

Keywords: Double framed soft fuzzy set, Double framed T(B)-soft fuzzy -algebra(ideal), doubt double framed T-soft fuzzy - algebra, doubt double framed T-soft fuzzy -ideal.

\section{Introduction}

In our daily life, the graph of uncertainty is very high in different fields like engineering, economics and in decision making problem. If we discussed about the classical mathematical tool which dealt with uncertainty is crisp set theory but with the passage of time uncertainty graph is increased. Then crisp theory [1] was not enough to handle this problem. At last fuzzy theory is introduced by L. A. Zadeh [2] in 1965 to handle vague concepts. This theory solved this problem and then it is used in many fields of our life like engineering, pharmacology, medical application and economics, among others. This is also affected by uncertainty because this concept based on membership function. We know that, sometime we feel hesitation to give membership value to any element which belong to fuzzy set. To overcome this problem, in 1999 Moldstov [3] introduced new concept which is known as soft set theory. He defined its applications in different areas like smoothness of function, measure integration, Riemannian integration, game theory and economics, among others.

After introduction of fuzzy theory and soft theory, now we discussed about the work of those researchers whose accepted this challenging theory and applied this concept in different algebraic structures like first time, Maji [4, 5] discussed this concept in decision making problem and combined the both concepts fuzzy set and soft set, and investigated their properties by giving examples. Cagman et al. [6] initiated to introduce this concept in group theory and discussed comparison between soft set, fuzzy set and rough sets and investigated its properties. Ali et.al [7] introduced some new operations on soft sets. These contradicted the some operations which are defined by Maji [5]. Jun and Ahn [8] initiated to introduce the concept of double framed soft and defined its notions. Further, they also discussed the product of double framed soft BCI/BCK-algebras. Furthermore, they studied the concept of double framed soft near ring. They also 
discussed major results related to this concept with the support of examples [9]. Naz [10] defined some operations on double framed double soft sets and introduced its notions. She also studied properties of BCI/BCK-algebras with the help of basic operations of double framed soft sets. Hadipour [11] applied the concept of double framed soft set in BF-algebras. Also the notations of double framed BF-algebras are investigated by examples. Shabir and Samreena [12], worked on double framed soft topological spaces and defined its notations. Bilal and Mahmood [13] initiated to introduce the concept of double framed T-soft fuzzy set and then concept is applied in BCI/BCI-algebra and investigated its properties to understand this concept. Further, they also discussed its union-product and defined its notions. Barbhuiya [14] introduced the doubt fuzzy ideal of BF-algebra and studied its properties like the major result in this article, Cartesian product of doubt fuzzy ideals also a doubt fuzzy ideal. For further information, we refer to reader papers [15-25] regarding soft and fuzzy algebraic structures.

In this paper, we applied the concept of double framed T-soft fuzzy set in BF-algebra to introduce DFSF-algebra, DFSF-deal, DDFSF-algebra and DDFSF-deal. Furthermore, we discussed union-product, intersection-product in DFSF-deal and DDFSF-deal, respectively.

\section{Preliminaries}

Let $X$ be a non-empty set. Then $A=\left\{\left\langle\sigma, \mu_{A}(\sigma)\right\rangle \mid \sigma \in X\right\}$ is called fuzzy set, where $\mu_{A}$ is a membership function which map each element of $X$ in $[0,1]$. Here we say that $A$ is fuzzy subset of $X$. The set of all fuzzy subsets of a set $X$ is denoted by $F P(X)$. Two fuzzy sets $A$ and $D$ are equal if and only if $\mu_{A}(\sigma)=\mu_{D}(\sigma)$ for all $\sigma \in X$. Where $\mu_{A}$ and $\mu_{D}$ are membership functions which map each element of $X$ in $[0,1]$.

A pair $(\mathscr{T}, E)$ is indicated to be soft set over if and only if $\mathscr{T}$ is a mapping from $E$ to the all subset of $U$ e.g

$$
(\mathscr{T}, E)=\{(e, \mathscr{T}(e)) \mid e \in E \text { and } \mathscr{T}(e) \in P(U)\}
$$

Where $P(U)$ is power set of $U$ and $\mathscr{T}: E \rightarrow P(U)$. The function $\mathscr{T}$ is an approximation function of the soft set $(\mathscr{T}, E)$. It is easy to see that soft set is parameterized family of subsets of $U$.The set of all soft sets over $U$ is denoted by $S(U)$.

A non-empty set $X$ under binary operation " $\star$ " with constant $\Theta$ is called a BF-algebra of type $(2, \Theta)$ is denoted by $(X, \star, \Theta)$ and defined as

$$
\text { for all } \sigma, \eta \in X\left\{\begin{array}{l}
\sigma \star \sigma=\Theta \\
\sigma \star \Theta=\sigma \\
\Theta \star(\sigma \star \eta)=\eta \star \sigma .
\end{array}\right.
$$

Definition 1. [8]. A double framed pair $(\mathscr{T}, \mathfrak{G})$ of $X$ over $U$ is said to double framed soft set, where $(\mathscr{T}, \mathfrak{G})$ both are soft sets over the same universe $U$ and denoted by $((\mathscr{T}, \mathfrak{G}), X)$ over $U$.

A $((\mathscr{T}, \mathfrak{G}), X)$ over $U$ is said to be double framed soft BCI/BCK-algebra if it satisfies $\mathscr{T}(\sigma \star \eta) \geq \mathscr{T}(\sigma) \cap \mathscr{T}(\eta)$ and $\mathfrak{G}(\sigma \star \eta) \subseteq \mathfrak{G}(\sigma) \cup \mathfrak{G}(\eta)$ for all $\sigma, \eta \in A$.

Definition 2. [13]. A double framed pair $(\mathscr{T}, \mu)$ of $X$ over $(U,[0,1])$ is said to DF-soft fuzzy set, where in $(\mathscr{T}, \mu), \mathscr{T}$ is a soft set over $U, \mu$ is fuzzy set over $[0,1]$ and denoted by $((\mathscr{T}, \mu), X)$ over $(U,[0,1])$.

Definition 3. [13]. Let $((\mathscr{T}, \mu), A)$ and $((\mathfrak{G}, g), D)$ be two "DF-soft fuzzy set" over $(U,[0,1])$ then $((\mathscr{T}, \mu), A)$ is said to be DF-soft fuzzy subset of $((\mathfrak{G}, g), D)$ if

(1) $A \subseteq D$, 
(2) $\mathscr{T}(a) \subseteq \mathfrak{G}(a)$ and $\mu(a) \geq g(a)$ for all $a \in A$.

We can write $((\mathscr{T}, \mu), A) \widetilde{\widetilde{C}}((\mathfrak{G}, g), D)$. In case $((\mathscr{T}, \mu), A)$ is super set of $((\mathfrak{G}, g), D)$.

Definition 4. [13]. Let $((\mathscr{T}, \mu), V)$ and $((\mathfrak{G}, g), D)$ are two "DF-soft fuzzy set" over $(U,[0,1])$. Then extended uniint of $((\mathscr{T}, \mu), V)$ and $((\mathfrak{G}, g), D)$ is defined as a "DF-SS" $((\mathscr{T} \widetilde{\cup} \mathfrak{G}, \mu \bar{\cap} g), V \cup D)$, where $\mathscr{T} \widetilde{\cup}:(V \cup D) \longrightarrow$ $P(U)$ defined by

$$
p \longrightarrow\left\{\begin{array}{c}
\mathscr{T}(p) \text { if } p \in V-D \\
\mathfrak{G}(p) \text { if } p \in D-V \\
\mathscr{T}(p) \cup \mathfrak{G}(p) \text { if } p \in V \cap D
\end{array}\right.
$$

and $\mu \bar{\cap} g:(V \cup D) \longrightarrow[0,1]$ defined by

$$
p \longrightarrow\left\{\begin{array}{c}
\mu(p) \text { if } p \in V-D \\
g(p) \text { if } p \in D-V \\
\mu(p) \wedge g(p) \text { if } p \in V \cap D
\end{array}\right.
$$

It is denoted by $((\mathscr{T}, \mu), V) \square_{\mathscr{E}}((\mathfrak{G}, g), D)=((\mathscr{T} \widetilde{\cup} \mathfrak{G}, \mu \bar{\cap} g), V \cup D)$. We shall call this uni-int "DF-soft fuzzy set" over $(U,[0,1])$ as union of "DF-soft fuzzy set" over $(U,[0,1])$.

Definition 5. [13]. Let $((\mathscr{T}, \mu), V)$ and $((\mathfrak{G}, g), D)$ are two "DFSFS" over $(U,[0,1])$. Then extended int-uni of $((\mathscr{T}, \mu), V)$ and $((\mathfrak{G}, g), D)$ is defined as a "DFSFS" $((\mathscr{T} \tilde{\cap} \mathfrak{G}, \mu \amalg g), V \cup D)$ over $(U,[0,1])$, where $\mathscr{T} \widetilde{\cap} \mathfrak{G}$ : $(V \cup D) \longrightarrow P(U)$ defined by

$$
p \longrightarrow\left\{\begin{array}{c}
\mathscr{T}(p) \text { if } p \in V-D \\
\mathfrak{G}(p) \text { if } p \in D-V \\
\mathscr{T}(p) \cap \mathfrak{G}(p) \text { if } p \in V \cap D
\end{array}\right.
$$

and $\mu \varpi g:(V \cup D) \longrightarrow[0,1]$ defined by

$$
p \rightarrow\left\{\begin{array}{c}
\mu(p) \text { if } p \in V-D \\
g(p) \text { if } p \in D-V \\
\mu(p) \vee g(p) \text { if } p \in V \cap D
\end{array}\right.
$$

It is denoted by $((\mathscr{T}, \mu), V) \overline{\Pi_{\mathscr{E}}}((\mathfrak{G}, g), D)=((\mathscr{T} \widetilde{\cap} \mathfrak{G}, \mu \bar{\cup} g), V \cup D)$. We shall call this int-uni "DF-soft fuzzy set” over $(U,[0,1])$ as intersection of DF- soft fuzzy set over $(U,[0,1])$.

Definition 6. [13]. Let $((\mathscr{T}, \mu), A)$ and $((\mathscr{T}, \mu), D)$ be "DF-soft fuzzy set" over $(U,[0,1])$. Then their product is defined as

$$
\begin{gathered}
\mathscr{T}_{A \vee D}: A \times D \longrightarrow P(U), \\
\mu_{A \wedge D}: A \times D \longrightarrow[0,1]
\end{gathered}
$$

such that $(\sigma, \eta) \longrightarrow\left\{\begin{array}{c}\mathscr{T}(\sigma) \cup \mathscr{T}(\eta) \\ \mu(\sigma) \wedge \mu(\eta)\end{array}\right.$, and denoted by $\left(\left(\mathscr{T}_{\vee}, \mu_{\wedge}\right), A \times D\right)$

\section{Related results}

This section is divided in to two sub sections; we proposed the concept of DFSF-algebra, DFSF-deal in $1^{s t}$ and in $2^{\text {nd }}$, DFSF-algebra and DFSF-deal. Also, we investigated its properties with the support of examples.

For brevity we also call $X$ a BF-algebra. A binary relation ' $\leq$ ' on $X$ can be defined by $\sigma \leq \eta$ if and only if $\sigma \star \eta=\Theta$ 


\subsection{Double framed B(T)-soft Fuzzy-algebra and double framed B(T)-soft Fuzzy-ideals}

In this subsection, we proposed the concept of DF-soft fuzzy-algebra and DF-soft fuzzy-ideal.

Definition 7. A DF-soft fuzzy set $((\mathscr{T}, \mu), X)$ over $(U,[0,1])$ is said to be double framed B-soft fuzzy algebra of BFalgebra $X$ if for all $\sigma, \eta \in X\left\{\begin{array}{c}\mathscr{T}(\sigma \star \eta) \subseteq \mathscr{T}(\sigma) \cup \mathscr{T}(\eta) \\ \mu(\sigma \star \eta) \geq \mu(\sigma) \cdot \mu(\eta) .\end{array}\right.$

Example 1. Let $U=\left\{\sigma_{1}, \sigma_{2}, \sigma_{3}, \sigma_{4}\right\}$ be an initial universe set and $X=\left\{p_{\Theta}, p_{2}, p_{3}, p_{4}\right\}$ be a set of parameters with following binary operation:

\begin{tabular}{|l|l|l|l|l|}
\hline$\star$ & $p_{\Theta}$ & $p_{2}$ & $p_{3}$ & $p_{4}$ \\
\hline$p_{\Theta}$ & $p_{\Theta}$ & $p_{2}$ & $p_{3}$ & $p_{4}$ \\
\hline$p_{2}$ & $p_{2}$ & $p_{\Theta}$ & $p_{2}$ & $p_{2}$ \\
\hline$p_{3}$ & $p_{3}$ & $p_{2}$ & $p_{\Theta}$ & $p_{2}$ \\
\hline$p_{4}$ & $p_{4}$ & $p_{2}$ & $p_{2}$ & $p_{\Theta}$ \\
\hline
\end{tabular}

Then $\left(X, \star, p_{a}\right)$ is a BF-algebra. Consider the DF-fuzzy soft set as follows:

$$
\begin{gathered}
\mathscr{T}: X \rightarrow P(U) \\
e \mapsto\left\{\begin{array}{cc}
\left\{\sigma_{1}, \sigma_{2}\right\} & \text { if } e \in\left\{p_{\Theta}, p_{2}\right\} \\
U & \text { if } e \in\left\{p_{3}, p_{4}\right\}
\end{array}\right.
\end{gathered}
$$

and $\mu: X \rightarrow[0,1]$

$$
e \mapsto\left\{\begin{array}{r}
.5 \text { if } e \in\left\{p_{\Theta}, p_{2}\right\} \\
.7 \text { if } e \in\left\{p_{3}, p_{4}\right\}
\end{array}\right.
$$

It is routine to verify that $((\mathscr{T}, \mu), X)$ over $(U,[0,1])$ is a double framed B-soft fuzzy BF-algebra.

Definition 8. A DF-soft fuzzy set $((\mathscr{T}, \mu), X)$ over $(U,[0,1])$ is said to be DFB-soft fuzzy sub algebra of BF-algebra X if for all $\sigma, \eta \in X$

$$
\left\{\begin{array}{c}
\mathscr{T}(\sigma \star \eta) \subseteq \mathscr{T}(\sigma) \cup \mathscr{T}(\eta) \\
\mu(\sigma \star \eta) \geq \mu(\sigma) \cdot \mu(\eta)
\end{array}\right.
$$

Definition 9. A DF-soft fuzzy set $((\mathscr{T}, \mu), X)$ over $(U,[0,1])$ is said to be double framed B-soft fuzzy ideal of $X$ if for all $\sigma, \eta \in X$

$$
\left\{\begin{array}{c}
\mathscr{T}(\Theta) \subseteq \mathscr{T}(\sigma) \text { and } \mathscr{T}(\sigma) \subseteq \mathscr{T}(\sigma \star \eta) \cup \mathscr{T}(\eta) \\
\mu(\Theta) \geq \mu(\sigma)^{2} \text { and } \mu(\sigma) \geq \mu(\sigma \star \eta) \mu(\eta)
\end{array}\right.
$$

In Example 1, if we take

$$
\mathscr{T}: X \rightarrow P(U) e \mapsto\left\{\begin{array}{l}
\left\{\sigma_{2}\right\} \quad \text { if } e \in\left\{p_{\Theta}, p_{2}\right\} \\
\left\{\sigma_{1}, \sigma_{2}\right\} \text { if } e \in\left\{p_{3}, p_{4}\right\}
\end{array}\right.
$$

And $\mu: X \rightarrow[0,1] e \mapsto\left\{\begin{array}{c}0.4 \text { if } e \in\left\{p_{\Theta}, p_{2}\right\} \\ 0.6 \text { if } e \in\left\{p_{3}, p_{4}\right\}\end{array} . \quad\right.$ Then $((\mathscr{T}, \mu), X)$ is a subalgebra. We note that $((\mathscr{T}, \mu), X)$ over $(U,[0,1])$ is also a DFB-soft fuzzy ideal. 
Definition 10. A DF-soft fuzzy set $((\mathscr{T}, \mu), X)$ over $(U,[0,1])$ is said to be DFT-soft fuzzy algebra of BF-algebra X iffor all $\sigma, \eta \in X$

$$
\left\{\begin{array}{l}
\mathscr{T}(\sigma \star \eta) \subseteq \mathscr{T}(\sigma) \cup \mathscr{T}(\eta) \\
\mu(\sigma \star \eta) \geq \mu(\sigma) \wedge \mu(\eta)
\end{array}\right.
$$

Example 2. Let $U=\left\{\sigma_{1}, \sigma_{2}, \sigma_{3}\right\}$ be an initial universe set and $X=\left\{p_{\Theta}, p_{b}, p_{c}\right\}$ be a set of parameters with following binary operation:

\begin{tabular}{|l|l|l|l|}
\hline$\star$ & $p_{\Theta}$ & $p_{b}$ & $p_{c}$ \\
\hline$p_{\Theta}$ & $p_{\Theta}$ & $p_{b}$ & $p_{c}$ \\
\hline$p_{b}$ & $p_{b}$ & $p_{\Theta}$ & $p_{\Theta}$ \\
\hline & & & \\
$p_{c}$ & $p_{c}$ & $p_{\Theta}$ & $p_{\Theta}$ \\
\hline
\end{tabular}

Then $\left(X, \star, p_{\Theta}\right)$ is a BF-algebra. Consider the DF-fuzzy soft set as follows:

$$
\begin{gathered}
\mathscr{T}: X \rightarrow P(U) \\
e \mapsto\left\{\begin{array}{rl}
\left\{\sigma_{1}\right\} & \text { if } e=p_{\Theta} \\
\left\{\sigma_{1}, \sigma_{2}\right\} & \text { if } e \in\left\{p_{b}, p_{c}\right\}
\end{array} .\right.
\end{gathered}
$$

And $\mu: X \rightarrow[0,1]$

$$
e \mapsto\left\{\begin{array}{l}
.5 \text { if } e=p_{\Theta} \\
.4 \text { if } e \in\left\{p_{b}, p_{c}\right\}
\end{array}\right.
$$

It is easy to see that $((\mathscr{T}, \mu), X)$ over $(U,[0,1])$ is a DFT-soft fuzzy BF-algebra. Note that, it is easy to see that all results which are proved in article [13] are satisfied if we replace $X$ (BCI/BCK-algebra) as a BF-algebra than BCI/BCK algebra.

Definition 11. A DF-soft fuzzy set $((\mathscr{T}, \mu), X)$ over $(U,[0,1])$ is said to be DFT-soft fuzzy sub algebra of BF-algebra X iffor all $\sigma, \eta \in X$

$$
\left\{\begin{array}{l}
\mathscr{T}(\sigma \star \eta) \subseteq \mathscr{T}(\sigma) \cup \mathscr{T}(\eta) \\
\mu(\sigma \star \eta) \geq \mu(\sigma) \wedge \mu(\eta)
\end{array}\right.
$$

Definition 12. A DF-soft fuzzy set $((\mathscr{T}, \mu), X)$ over $(U,[0,1])$ is said to be DFT-soft fuzzy ideal of $X$ if for all $\sigma, \eta \in X$

$$
\left\{\begin{array}{c}
\mathscr{T}(\Theta) \subseteq \mathscr{T}(\sigma) \text { and } \mathscr{T}(\sigma) \subseteq \mathscr{T}(\sigma \star \eta) \cup \mathscr{T}(\eta) \\
\mu(\Theta) \geq \mu(\sigma) \text { and } \mu(\sigma) \geq \mu(\sigma \star \eta) \wedge \mu(\eta)
\end{array}\right.
$$

Proposition 1. If $((\mathscr{T}, \mu), X)$ over $(U,[0,1])$ be a DFT-soft fuzzy ideal of $X$ then following hold,

(a) if $\sigma \leq \eta$ then $\left\{\begin{array}{l}\mathscr{T}(\sigma) \subseteq \mathscr{T}(\eta) \\ \mu(\sigma) \geq \mu(\eta),\end{array}\right.$

(b) if $\mathscr{T}(\sigma \star \eta)=\mathscr{T}(\Theta)$ and $\mu(\sigma \star \eta)=\mu(\Theta)$ then $\left\{\begin{array}{l}\mathscr{T}(\sigma) \subseteq \mathscr{T}(\eta) \\ \mu(\sigma) \geq \mu(\eta)\end{array}\right.$

(c) if $\sigma \star \eta \leq w$ then $\left\{\begin{array}{l}\mathscr{T}(\sigma) \subseteq \mathscr{T}(\eta) \cup \mathscr{T}(w) \\ \mu(\sigma) \geq \mu(\eta) \wedge \mathscr{T}(w),\end{array}\right.$

For all $\sigma, \eta, w \in X$. 
Proof. (a) Let $\sigma \leq \eta$ then $\sigma \star \eta=\Theta$. Now by definition of $((\mathscr{T}, \mu), X)$ such that $\mathscr{T}(\sigma) \supseteq \mathscr{T}(\sigma \star \eta) \cup \mathscr{T}(\eta)$ and $\mu(\sigma) \geq \mu(\sigma \star \eta) \wedge \mu(\eta)$ then $\mathscr{T}(\sigma) \subseteq \mathscr{T}(\Theta) \cup \mathscr{T}(\eta), \mu(\sigma) \geq \mu(\Theta) \wedge \mu(\eta)$ implies that $\mathscr{T}(\sigma) \subseteq \mathscr{T}(\eta)$ and $\mu(\sigma) \geq \mu(\eta)$.

(b) Let $\mathscr{T}(\sigma \star \eta)=\mathscr{T}(\Theta)$ and $\mu(\sigma \star \eta)=\mu(\Theta)$. Then by definition of $(\mathscr{T}, \mu), X)$, we have $\mathscr{T}(\sigma) \subseteq \mathscr{T}(\sigma \star \eta) \cup \mathscr{T}(\eta)$ and $\mu(\sigma) \geq \mu(\sigma \star \eta) \wedge \mu(\eta)$ implies that $\mathscr{T}(\sigma) \subseteq \mathscr{T}(\Theta) \cup \mathscr{T}(\eta), \mu(\sigma) \geq \mu(\Theta) \wedge \mu(\eta)$ implies that $\mathscr{T}(\sigma) \subseteq \mathscr{T}(\eta)$ and $\mu(\sigma) \geq \mu(\eta)$. (c) By definition of $((\mathscr{T}, \mu), X)$, we have

$$
\mathscr{T}(\sigma) \subseteq \mathscr{T}(\sigma \star \eta) \cup \mathscr{T}(\eta) \quad \text { and } \quad \mu(\sigma) \geq \mu(\sigma \star \eta) \wedge \mu(\eta) \ldots
$$

Let $\sigma \star \eta \leq w$ then $(\sigma \star \eta) \star w=\Theta, \mathscr{T}(\sigma \star \eta) \subseteq w$ and $\mu(\sigma \star \eta) \geq \mu(w)$. Now by following (1), we have

$\mathscr{T}(\sigma \star \eta) \subseteq \mathscr{T}((\sigma \star \eta) \star w) \cup \mathscr{T}(w)$ and $\mu(\sigma \star \eta) \geq \mu((\sigma \star \eta) \star w) \wedge \mu(w)$

Implies $\mathscr{T}(\sigma \star \eta) \subseteq \mathscr{T}(\Theta) \cup \mathscr{T}(w)$ and $\mu(\sigma \star \eta) \geq \mu(\Theta) \wedge \mu(w)$

Implies $\mathscr{T}(\sigma \star \eta) \subseteq \mathscr{T}(w)$ and $\mu(\sigma \star \eta) \geq \mu(w)$

Implies $\mathscr{T}(\sigma \star \eta) \cup \mathscr{T}(\eta) \subseteq \mathscr{T}(\eta) \cup \mathscr{T}(w) \quad$ and $\quad \mu(\sigma \star \eta) \wedge \mu(\eta) \geq \mu(w) \wedge \mu(\eta)$.

From (1) and (2), we have $\mathscr{T}(\sigma) \subseteq \mathscr{T}(\eta) \cup \mathscr{T}(w)$ and $\mu(\sigma) \geq \mu(\eta) \wedge \mathscr{T}(w)$.

Theorem 1. Let $((\mathscr{T}, \mu), X)$ and $((\mathfrak{G}, g), X)$ over $(U,[0,1])$ be two DFT-soft fuzzy ideals of $X$ then prove that their intersection is also a DFT-soft fuzzy ideal of X.

Proof. Let $((\mathscr{T}, \mu), X)$ and $((\mathfrak{G}, g), X)$ over $(U,[0,1])$ be two DF-soft fuzzy ideals of $X$ and $\sigma, \eta \in X$ then $\left\{\begin{array}{c}\mathscr{T}(\Theta) \subseteq \mathscr{T}(\sigma) \text { and } \mathscr{T}(\sigma) \subseteq \mathscr{T}(\sigma \star \eta) \cup \mathscr{T}(\eta) \\ \mu(\Theta) \geq \mu(\sigma) \text { and } \mu(\sigma) \geq \mu(\sigma \star \eta) \wedge \mu(\eta)\end{array}\right.$ and $\left\{\begin{array}{c}\mathfrak{G}(\Theta) \subseteq \mathfrak{G}(\sigma) \text { and } \mathfrak{G}(\sigma) \subseteq \mathfrak{G}(\sigma \star \eta) \cup \mathfrak{G}(\eta) \\ g(\Theta) \geq g(\sigma) \text { and } g(\sigma) \geq g(\sigma \star \eta) \wedge g(\eta)\end{array}\right.$

Now, union of $((\mathscr{T}, \mu), X)$ and $((\mathfrak{G}, g), X)$ is defined as $((\mathscr{T}, \mu), X) \sqcup_{\mathscr{E}}((\mathfrak{G}, g), X)=((H, h), X)$ where $H=\mathscr{T} \widetilde{\cup} \mathfrak{G}$ and $h=\mu \bar{\cap} g$.

It is given that $\mathscr{T}(\Theta) \subseteq \mathscr{T}(\sigma), \mu(\Theta) \geq \mu(\sigma)$ and $\mathfrak{G}(\Theta) \subseteq \mathfrak{G}(\sigma), g(\Theta) \geq g(\sigma)$ then

$\mathscr{T}(\Theta) \cup \mathfrak{G}(\Theta) \subseteq \mathscr{T}(\sigma) \cup \mathfrak{G}(\sigma)$ and $\mu(\Theta) \wedge g(\Theta) \geq \mu(\sigma) \wedge g(\sigma)$

$\Longrightarrow \mathscr{T}(\Theta) \cup \mathfrak{G}(\Theta) \subseteq \mathscr{T}(\sigma) \cup \mathfrak{G}(\Theta)$ and $\mu(\Theta) \wedge g(\Theta) \geq \mu(\sigma) \wedge g(\sigma)$

$\Longrightarrow(\mathscr{T} \widetilde{\cup} \mathfrak{G})(\Theta) \subseteq(\mathscr{T} \widetilde{\cup} \mathfrak{G})(\sigma)$ and $(\mu \bar{\cap} g)(\Theta) \geq(\mu \bar{\cap} g)(\sigma)$

Implies $H(\Theta) \subseteq H(\sigma)$ and $h(\Theta) \geq h(\sigma)$.

Further, $\mathscr{T}(\sigma) \subseteq \mathscr{T}(\sigma \star \eta) \cup \mathscr{T}(\eta), \quad \mu(\sigma) \geq \mu(\sigma \star \eta) \wedge \mu(\eta) \quad$ and $\quad \mathfrak{G}(\sigma) \subseteq \mathfrak{G}(\sigma \star \eta) \cup \mathfrak{G}(\eta)$, $g(\sigma) \geq g(\sigma \star \eta) \wedge g(\eta)$, we

$\mathscr{T}(\sigma) \cup \mathfrak{G}(\sigma) \subseteq(\mathscr{T}(\sigma \star \eta) \cup \mathscr{T}(\eta)) \cup(\mathfrak{G}(\sigma \star \eta) \cup \mathfrak{G}(\eta)) \quad$ and $\quad \mu(\sigma) \wedge g(\sigma) \geq(\mu(\sigma \star \eta) \wedge \mu(\eta)) \wedge(g(\sigma \star \eta) \wedge g(\eta))$,

$\Longrightarrow \mathscr{T}(\sigma) \cup \mathfrak{G}(\sigma) \subseteq(\mathscr{T}(\sigma \star \eta) \cup \mathfrak{G}(\sigma \star \eta)) \cup(\mathfrak{G}(\eta) \cup \mathfrak{G}(\eta)) \quad$ and $\quad \mu(\sigma) \wedge g(\sigma) \geq(\mu(\sigma \star \eta) \wedge g(\sigma \star \eta)) \wedge(g(\eta) \wedge g(\eta))$, 


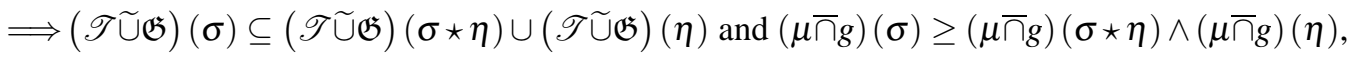

$\Longrightarrow H(\sigma) \subseteq H(\sigma \star \eta) \cup H(\eta)$ and $h(\sigma) \geq h(\sigma \star \eta) \wedge h(\eta)$.

Hence, result is proved.

Note that, intersection of $((\mathscr{T}, \mu), X)$ and $((\mathfrak{G}, g), X)$ over $(U,[0,1])$ is also said to be extended int-uni of $((\mathscr{T}, \mu), X)$ and $((\mathfrak{G}, g), X)$ and known as extended int-uni doubt double T-soft fuzzy ideal of $X$.

Corollary 1. Let $\left\{\left(\left(\mathscr{T}_{j}, \mu_{j}\right), X\right)\right.$ is the collection of ideals of $\left.X: j=1,2,3, \ldots\right\}$. Then their Intersection is also a DFTsoft fuzzy ideal of BF-algebra $X$.

Theorem 2. Let $\left(\left(\mathscr{T}_{1}, \mu_{1}\right), X\right)$ and $\left(\left(\mathscr{T}_{2}, \mu_{2}\right), X\right)$ be two DFT-soft fuzzy ideals of $X$ over $(U,[0,1])$ then prove that their union-product is also DFT-soft fuzzy ideal of $X \times X$.

Proof. Let for any $(\sigma, \eta),(y, z) \in X \times X$, we have

$$
\begin{aligned}
& \left(\mathscr{T}_{1} \vee \mathscr{T}_{2}\right)(\Theta, \Theta)=\mathscr{T}_{1}(\Theta) \cup \mathscr{T}_{2}(\Theta) \subseteq \mathscr{T}_{1}(\sigma) \cup \mathscr{T}_{2}(\eta)=\left(\mathscr{T}_{1} \vee \mathscr{T}_{2}\right)(\sigma, \eta) \\
& \Longrightarrow\left(\mathscr{T}_{1} \vee \mathscr{T}_{2}\right)(\Theta, \Theta) \subseteq\left(\mathscr{T}_{1} \vee \mathscr{T}_{2}\right)(\sigma, \eta)\left(\mu_{1} \wedge \mu_{2}\right)(\Theta, \Theta)=\mu_{1}(\Theta) \wedge \mu_{2}(\Theta) \geq \mu_{1}(\sigma) \wedge \mu_{2}(\eta) \\
& \Longrightarrow\left(\mu_{1} \wedge \mu_{2}\right)(\Theta, \Theta) \geq \mu_{1} \wedge \mu_{2}(\sigma, \eta) .
\end{aligned}
$$

Since $(\sigma, \eta),(y, z) \in X \times X$, we have $\left(\mathscr{T}_{1} \vee \mathscr{T}_{2}\right)(\sigma, \eta)=\mathscr{T}_{1}(\sigma) \cup \mathscr{T}_{2}(\eta)$. As $\left(\left(\mathscr{T}_{1}, \mu_{1}\right), X\right)$ and $\left(\left(\mathscr{T}_{2}, \mu_{2}\right), X\right)$ both are ideals so we have

$$
\begin{aligned}
& \left(\mathscr{T}_{1} \vee \mathscr{T}_{2}\right)(\sigma, \eta)=\mathscr{T}_{1}(\sigma) \cup \mathscr{T}_{2}(\eta) \\
& \subseteq\left(\mathscr{T}_{1}(\sigma \star y) \cup \mathscr{T}_{1}(y)\right) \cup\left(\mathscr{T}_{2}(\eta \star z) \cup \mathscr{T}_{2}(z)\right) \\
& =\left(\mathscr{T}_{1}(\sigma \star y) \cup \mathscr{T}_{2}(\eta \star z)\right) \cup\left(\mathscr{T}_{1}(y) \cup \mathscr{T}_{2}(z)\right) \\
& =\left(\left(\mathscr{T}_{1} \vee \mathscr{T}_{2}\right)(\sigma \star y, \eta \star z)\right) \cup\left(\left(\mathscr{T}_{1} \vee \mathscr{T}_{2}\right)(y, z)\right) \\
& =\left(\left(\mathscr{T}_{1} \vee \mathscr{T}_{2}\right)((\sigma, \eta) \star(y, z)) \cup\left(\left(\mathscr{T}_{1} \vee \mathscr{T}_{2}\right)(y, z)\right)\right. \\
& \Longrightarrow\left(\mathscr{T}_{1} \vee \mathscr{T}_{2}\right)(\sigma, \eta) \subseteq\left(\left(\mathscr{T}_{1} \vee \mathscr{T}_{2}\right)((\sigma, \eta) \star(y, z))\right) \cup\left(\left(\mathscr{T}_{1} \vee \mathscr{T}_{2}\right)(y, z)\right) \\
& \left(\mu_{1} \wedge \mu_{2}\right)(\sigma, \eta)=\mu_{1}(\sigma) \wedge \mu_{2}(\eta) \\
& \geq\left(\mu_{1}(\sigma \star y) \wedge \mu_{1}(y)\right) \wedge\left(\mu_{2}(\eta \star z) \wedge \mu_{2}(z)\right) \\
& =\left(\mu_{1}(\sigma \star y) \wedge \mu_{2}(\eta \star z)\right) \wedge\left(\mu_{1}(y) \wedge \mu_{2}(z)\right) \\
& =\left(\left(\mu_{1} \vee \mu_{2}\right)(\sigma \star y, \eta \star z)\right) \wedge\left(\left(\mu_{1} \vee \mu_{2}\right)(y, z)\right) \\
& =\left(\left(\mu_{1} \wedge \mu_{2}\right)((\sigma, \eta) \star(y, z))\right) \wedge\left(\left(\mu_{1} \wedge \mu_{2}\right)(y, z)\right) \\
& \Longrightarrow\left(\mu_{1} \wedge \mu_{2}\right)(\sigma, \eta) \geq\left(\left(\mu_{1} \wedge \mu_{2}\right)((\sigma, \eta) \star(y, z))\right) \wedge\left(\left(\mu_{1} \wedge \mu_{2}\right)(y, z)\right)
\end{aligned}
$$

Hence, $\left(\left(\mathscr{T}_{\vee}, \mu_{\wedge}\right), A \times D\right)$ is a "DFT-soft fuzzy ideal" over $(U,[0,1])$.

\subsection{Doubt double framed soft Fuzzy-algebras of BF or BCI/BCK-algebras}

In this subsection, we proposed the concept of doubt DF-soft fuzzy-algebra of BF or BCI/BCK-algebra and properties are investigated with the support of examples.

Note that in this section, we only proved results for BF-algebras. The results and definitions are also hold for doubt DF-soft fuzzy algebras of BCI/BCK-algebra which are prove for BF-algebra if we replace $X$ as a BCI/BCK-algebra. 
Definition 13. A DF-soft fuzzy set $((\mathscr{T}, \mu), X)$ over $(U,[0,1])$ is said to be doubt DF-soft fuzzy set if behaviour of both sets is changed for example, a DF-soft fuzzy set $((\mathscr{T}, \mu), X)$ over $(U,[0,1])$ is said to be doubt DF-soft fuzzy algebra (DDF-soft fuzzy algebra) of BF-algebra $X$ if for all $\sigma, \eta \in X$

$$
\left\{\begin{array}{l}
\mathscr{T}(\sigma \star \eta) \supseteq \mathscr{T}(\sigma) \cap \mathscr{T}(\eta) \\
\mu(\sigma \star \eta) \leq \mu(\sigma) \vee \mu(\eta)
\end{array}\right.
$$

Lemma 1. Every “DDF-soft fuzzy algebra” $((\mathscr{T}, \mu), \delta)$ over $(U,[0,1])$ satisfies the following conditions

$$
\mathscr{T}(\Theta) \supseteq \mathscr{T}(\sigma) \text { and } \mu(\Theta) \leq \mu(\sigma) \text { for all } \sigma \in \delta \text {. }
$$

Proof. By definition of $((\mathscr{T}, \mu), \delta)$, we have $\mathscr{T}(\sigma \star \sigma) \supseteq \mathscr{T}(\sigma) \cap \mathscr{T}(\sigma)$ for all $\sigma \in \delta$ implies $\mathscr{T}(\sigma \star \sigma)=\mathscr{T}(\Theta) \supseteq \mathscr{T}(\sigma)$ because $\sigma \star \sigma=\Theta$.

Now $\mu(\sigma \star \sigma) \leq \mu(\sigma) \vee \mu(\sigma)$ for all $\sigma \in \delta$. Since $\sigma \star \sigma=\Theta$ so $\mu(\sigma \star \sigma)=\mu(\Theta) \leq \mu(\sigma)$.

Hence, proof is complete.

Theorem 3. For a "DDF-soft fuzzy algebra" $((\mathscr{T}, \mu), \delta)$ over $(U,[0,1])$, the following are equivalent;

$$
\begin{gathered}
(1) \mathscr{T}(\Theta)=\mathscr{T}(\sigma) \text { and } \mu(\Theta)=\mu(\sigma) \text { for all } \sigma \in \delta, \\
\mathscr{T}(\sigma \star \eta) \supseteq \mathscr{T}(\eta) \text { and } \mu(\sigma \star \eta) \leq \mu(\eta) \text { for all } \sigma, \eta \in \delta .
\end{gathered}
$$

Proof. $(1) \Rightarrow(2)$, We suppose that (1) is valid. Now by definition of $((\mathscr{T}, \mu), \delta)$, we have (for all $\sigma \in \delta) \mathscr{T}(\sigma \star \eta) \supseteq$ $\mathscr{T}(\sigma) \cap \mathscr{T}(\eta)=\mathscr{T}(\Theta) \cap \mathscr{T}(\eta)=\mathscr{T}(\eta)$ because $\mathscr{T}(\Theta)=\mathscr{T}(\sigma)$ for all $\sigma \in \delta$, now (for all $\sigma \in \delta) \mu(\sigma \star \eta) \leq \mu(\sigma) \vee$ $\mu(\eta)=\mu(\Theta) \vee \mu(\eta)=\mu(\eta)$ because $\mu(\Theta)=\mu(\sigma)$ for all $\sigma \in \delta$. (2) $\Rightarrow$ (1), Assume that (2) is valid. Then for $\eta=\Theta$ such that $\mathscr{T}(\sigma \star \Theta) \supseteq \mathscr{T}(\Theta)$ and $\mu(\sigma \star \Theta) \leq \mu(\Theta)$ implies $\mathscr{T}(\sigma) \supseteq \mathscr{T}(\Theta)$ and $\mu(\sigma) \leq \mu(\Theta)$ because $\sigma \star \Theta=\sigma$, then by 3.8. Lemma we have, $\mathscr{T}(\Theta) \supseteq \mathscr{T}(\sigma)$ and $\mu(\Theta) \leq \mu(\sigma)$ for all $\sigma \in \delta$, so $\mathscr{T}(\sigma)=\mathscr{T}(\Theta)$ and $\mu(\sigma)=\mu(\Theta)$.

Theorem 4. The extended int-uni of "DDF-soft fuzzy set" over $(U,[0,1])$ of two doubt double-framed soft fuzzy algebras $((\mathscr{T}, \mu), X)$ and $((\mathfrak{G}, g), X)$ over $(U,[0,1])$ is "DDF-soft fuzzy algebra” over $(U,[0,1])$.

Proof. Let $\sigma, \eta \in X$ then we have

$$
\begin{aligned}
(\mathscr{T} \widetilde{G})(\sigma \star \eta) & =\mathscr{T}(\sigma \star \eta) \cap \mathfrak{G}(\sigma \star \eta) \supseteq(\mathscr{T}(\sigma) \cap \mathscr{T}(\eta)) \cap(\mathfrak{G}(\sigma) \cap \mathfrak{G}(\eta)) \\
& =(\mathscr{T}(\sigma) \cap \mathfrak{G}(\sigma)) \cap(\mathscr{T}(\eta) \cap \mathfrak{G}(\eta))=(\mathscr{T} \widetilde{G})(\sigma) \cap(\mathscr{T} \widetilde{G})(\eta), \\
(\mu \varpi g)(\sigma \star \eta) & =\mu(\sigma \star \eta) \vee g(\sigma \star \eta) \leq(\mu(\sigma) \vee \mu(\eta)) \vee(g(\sigma) \wedge g(\eta)) \\
& =(\mu(\sigma) \vee g(\sigma)) \vee(\mu(\eta) \vee g(\eta))=(\mu \amalg g)(\sigma) \vee(\mu \varpi g)(\eta) .
\end{aligned}
$$

Hence, $V_{(\mathscr{T}, \mu)} \overline{\Pi_{\mathscr{E}}} D_{(\mathfrak{G}, g)}$ is a "DDF-soft fuzzy algebra" over $(U,[0,1])$.

Theorem 5. The extended uni-int of two doubt double-framed soft fuzzy algebras $((\mathscr{T}, \mu), X)$ and $((\mathfrak{G}, g), X)$ over $(U,[0,1])$ is ”DDF-soft fuzzy algebra” over $(U,[0,1])$ if $((\mathscr{T}, \mu), X) \stackrel{\widetilde{C}}{\stackrel{C}{C}}((\mathfrak{G}, g), X)$. 
Proof. Let $\sigma, \eta \in X$ then we have

$$
\begin{aligned}
(\mathscr{T} \widetilde{\cup} \mathfrak{G})(\sigma \star \eta) & =\mathscr{T}(\sigma \star \eta) \cup \mathfrak{G}(\sigma \star \eta)=\mathfrak{G}(\sigma \star \eta) \supseteq \mathfrak{G}(\sigma) \cap \mathfrak{G}(\eta) \\
& =(\mathscr{T}(\sigma) \cap \mathfrak{G}(\sigma)) \cap(\mathscr{T}(\eta) \cap \mathfrak{G}(\eta))=(\mathscr{T} \widetilde{\cup} \mathfrak{G})(\sigma) \cup(\mathscr{T} \widetilde{\cup} \mathfrak{G})(\eta), \\
(\mu \bar{\cap} g)(\sigma \star \eta) & =\mu(\sigma \star \eta) \wedge g(\sigma \star \eta)=g(\sigma \star \eta) \leq g(\sigma) \vee g(\eta) \\
& =(\mu(\sigma) \vee g(\sigma)) \wedge(\mu(\eta) \vee g(\eta))=(\mu \bar{\cap} g)(\sigma) \wedge(\mu \bar{\cap} g)(\eta) .
\end{aligned}
$$

Hence, $V_{(\mathscr{T}, \mu)} \square_{\mathscr{E}} D_{(\mathfrak{G}, g)}$ is an extended uni-int "DDF-soft fuzzy algebra" over $(U,[0,1])$.

Definition 14. If $\left(\left(\mathscr{T}_{1}, \mu_{1}\right), C\right)$ and $\left(\left(\mathscr{T}_{2}, \mu_{2}\right), D\right)$ be two doubt DF-soft fuzzy ideal of $X$ over $(U,[0,1])$ the their intersection product is defined as $\left\{\begin{array}{l}\mathscr{T}_{1} \wedge \mathscr{T}_{2}: C \times D \longrightarrow P(U) \\ \mu_{1} \vee \mu_{2}: C \times D \longrightarrow[0,1]\end{array}\right.$, such that $(c, d) \longrightarrow\left\{\begin{array}{c}\mathscr{T}(c) \cap \mathscr{T}(d) \\ \mu(c) \vee \mu(d)\end{array}\right.$ And denoted by $\left(\left(\mathscr{T}_{\wedge}, \mu_{\vee}\right), C \times D\right)$.

Theorem 6. Let $((\mathscr{T}, \mu), C)$ and $((\mathscr{T}, \mu), D)$ be doubt DF-soft fuzzy algebras over $(U,[0,1])$. Then prove that $\left(\left(\mathscr{T}_{\wedge}, \mu_{\vee}\right), C \times D\right)$ is also a "DDF-soft fuzzy algebra" over $(U,[0,1])$.

Proof. We know that $\left(C \times D, \diamond,(\Theta, \Theta)\right.$ is also a BF-algebra. Then we only prove that $\left(\left(\mathscr{T}_{\wedge}, \mu_{\vee}\right), C \times D\right)$ is a "DDF-soft fuzzy algebra" over $(U,[0,1])$. Let $(x, y),(\sigma, \eta) \in C \times D$, we have

$$
\begin{aligned}
\mathscr{T}_{C \wedge D}((x, y) \diamond(\sigma, \eta)) & =\mathscr{T}_{C \wedge D}(x \star \sigma, y \star \eta) \\
& =\mathscr{T}(x \star \sigma) \cap \mathscr{T}(y \star \eta) \supseteq(\mathscr{T}(x) \cap \mathscr{T}(\sigma)) \cap(\mathscr{T}(y) \cap \mathscr{T}(\eta)) \\
& =(\mathscr{T}(x) \cap \mathscr{T}(y)) \cap(\mathscr{T}(\sigma) \cap \mathscr{T}(\eta)) \\
& =\mathscr{T}_{C \wedge D}(x, \sigma) \cap \mathscr{T}_{C \wedge D}(y, \eta) \\
\mu_{C \vee D}((x, y) \diamond(\sigma, \eta)) & =\mu_{C \vee D}(x \star \sigma, y \star \eta)=\mu(x \star \sigma) \vee \mu(y \star \eta) \\
& \leq(\mu(x) \vee \mu(\sigma)) \vee(\mu(y) \vee \mu(\eta)) \\
& =(\mu(x) \vee \mu(y)) \vee(\mu(\sigma) \vee \mu(\eta)) \\
& =\mu(x \star y) \vee \mu(\sigma \star \eta) \\
& =\mu_{C \vee D}(x, y) \vee \mu_{C \vee D}(\sigma, \eta) .
\end{aligned}
$$

Hence, $\left(\left(\mathscr{T}_{\wedge}, \mu_{\vee}\right), C \times D\right)$ is a "DDF-soft fuzzy algebra" over $(U,[0,1])$.

\subsubsection{Doubt double framed soft Fuzzy ideals of BF-algebras}

In this subsection, we proposed the concept of doubt DF-soft fuzzy ideals of BF-algebra.

Definition 15. A double framed pair $(\mathscr{T}, \mu)$ of $X$ over $(U,[0,1])$ is said to be doubt DF-soft fuzzy sub algebra of BFalgebra $X$ if for all $\sigma, \eta \in X$

$$
\left\{\begin{array}{l}
\mathscr{T}(\sigma \star \eta) \supseteq \mathscr{T}(\sigma) \cap \mathscr{T}(\eta) \\
\mu(\sigma \star \eta) \leq \mu(\sigma) \vee \mu(\eta)
\end{array}\right.
$$

Definition 16. A DF-soft fuzzy set $((\mathscr{T}, \mu), X)$ over $(U,[0,1])$ is said to be doubt DF-soft fuzzy ideal of $X$ if for all $\sigma, \eta \in X$

$$
\left\{\begin{array}{c}
\mathscr{T}(\Theta) \supseteq \mathscr{T}(\sigma) \quad \text { and } \quad \mathscr{T}(\sigma) \supseteq \mathscr{T}(\sigma \star \eta) \cap \mathscr{T}(\eta) \\
\mu(\Theta) \leq \mu(\sigma) \text { and } \quad \mu(\sigma) \leq \mu(\sigma \star \eta) \vee \mu(\eta)
\end{array}\right.
$$


Proposition 2. If $((\mathscr{T}, \mu), X)$ over $(U,[0,1])$ be a doubt DF-soft fuzzy ideal of $X$ then following hold,

(a) if $\sigma \leq \eta$ then $\left\{\begin{array}{l}\mathscr{T}(\sigma) \supseteq \mathscr{T}(\eta) \\ \mu(\sigma) \leq \mu(\eta)\end{array}\right.$

(b) if $\mathscr{T}(\sigma \star \eta)=\mathscr{T}(\Theta)$ and $\mu(\sigma \star \eta)=\mu(\Theta)$ then $\left\{\begin{array}{l}\mathscr{T}(\sigma) \supseteq \mathscr{T}(\eta) \\ \mu(\sigma) \leq \mu(\eta),\end{array}\right.$

(c) if $\sigma \star \eta \leq w$ then $\left\{\begin{array}{l}\mathscr{T}(\sigma) \supseteq \mathscr{T}(\eta) \cap \mathscr{T}(w) \\ \mu(\sigma) \leq \mu(\eta) \vee \mathscr{T}(w)\end{array}\right.$

For all $\sigma, \eta, w \in X$.

Proof. (a) Let $\sigma \leq \eta$ then $\sigma \star \eta=\Theta$. Now by definition of $((\mathscr{T}, \mu), X)$ such that $\mathscr{T}(\sigma) \supseteq \mathscr{T}(\sigma \star \eta) \cap \mathscr{T}(\eta)$ and $\mu(\sigma) \leq \mu(\sigma \star \eta) \vee \mu(\eta)$ then $\mathscr{T}(\sigma) \supseteq \mathscr{T}(\Theta) \cap \mathscr{T}(\eta), \mu(\sigma) \leq \mu(\Theta) \vee \mu(\eta)$ implies that $\mathscr{T}(\sigma) \supseteq \mathscr{T}(\eta)$ and $\mu(\sigma) \leq \mu(\eta)$.

(b) Let $\mathscr{T}(\sigma \star \eta)=\mathscr{T}(\Theta)($ and $\mu(\sigma \star \eta)=(\Theta)$. Then by definition of $(\mathscr{T}, \mu), X)$, we have $\mathscr{T}(\sigma) \supseteq \mathscr{T}(\sigma \star \eta) \cap \mathscr{T}(\eta)$ and $\mu(\sigma) \leq \mu(\sigma \star \eta) \vee \mu(\eta)$ implies that $\mathscr{T}(\sigma) \supseteq \mathscr{T}(\Theta) \cap \mathscr{T}(\eta), \mu(\sigma) \leq \mu(\Theta) \vee \mu(\eta)$ implies that $\mathscr{T}(\sigma) \supseteq \mathscr{T}(\eta)$ and $\mu(\sigma) \leq \mu(\eta)$.

(c) By definition of $((\mathscr{T}, \mu), X)$, we have

$$
\mathscr{T}(\sigma) \supseteq \mathscr{T}(\sigma \star \eta) \cap \mathscr{T}(\eta) \quad \text { and } \quad \mu(\sigma) \leq \mu(\sigma \star \eta) \vee \mu(\eta)
$$

Let $\sigma \star \eta \leq w$ then $(\sigma \star \eta) \star w=\Theta, \mathscr{T}(\sigma \star \eta) \supseteq w$ and $\mu(\sigma \star \eta) \leq \mu(w)$. Now by following (4), we have $\mathscr{T}(\sigma \star \eta) \supseteq \mathscr{T}((\sigma \star \eta) \star w) \cap \mathscr{T}(w) \quad$ and $\quad \mu(\sigma \star \eta) \leq \mu((\sigma \star \eta) \star w) \vee \mu(w)$

Implies $\mathscr{T}(\sigma \star \eta) \supseteq \mathscr{T}(\Theta) \cap \mathscr{T}(w) \quad$ and $\quad \mu(\sigma \star \eta) \leq \mu(\Theta) \vee \mu(w)$

Implies $\mathscr{T}(\sigma \star \eta) \supseteq \mathscr{T}(w)$ and $\mu(\sigma \star \eta) \leq \mu(w)$.

$$
\text { Implies } \mathscr{T}(\sigma \star \eta) \cap \mathscr{T}(\eta) \supseteq \mathscr{T}(\eta) \cap \mathscr{T}(w) \quad \text { and } \quad \mu(\sigma \star \eta) \vee \mu(\eta) \leq \mu(w) \vee \mu(\eta)
$$

From (4) and (5), we have $\mathscr{T}(\sigma) \supseteq \mathscr{T}(\eta) \cap \mathscr{T}(w)$ and $\mu(\sigma) \leq \mu(\eta) \vee \mathscr{T}(w)$

Theorem 7. Let $((\mathscr{T}, \mu), X)$ and $((\mathfrak{G}, g), X)$ over $(U,[0,1])$ be two doubt DF-soft fuzzy ideal of $X$ then prove that their intersection is also a doubt DF-soft fuzzy ideal of $X$.

Proof. Let $((\mathscr{T}, \mu), X)$ and $((\mathfrak{G}, g), X)$ over $(U,[0,1])$ be two doubt DF-soft fuzzy ideal of $X$ and $\sigma, \eta \in X$ then $\left\{\begin{array}{l}\mathscr{T}(\Theta) \supseteq \mathscr{T}(\sigma) \quad \text { and } \quad \mathscr{T}(\sigma) \supseteq \mathscr{T}(\sigma \star \eta) \cap \mathscr{T}(\eta) \\ \mu(\Theta) \leq \mu(\sigma) \quad \text { and } \quad \mu(\sigma) \leq \mu(\sigma \star \eta) \vee \mu(\eta) .\end{array} \quad\right.$ and $\quad\left\{\begin{array}{l}\mathfrak{G}(\Theta) \supseteq \mathfrak{G}(\sigma) \quad \text { and } \quad \mathfrak{G}(\sigma) \supseteq \mathfrak{G}(\sigma \star \eta) \cap \mathfrak{G}(\eta) \\ g(\Theta) \leq g(\sigma) \quad \text { and } \quad g(\sigma) \leq g(\sigma \star \eta) \vee g(\eta)\end{array}\right.$

Now, intersection of $((\mathscr{T}, \mu), X)$ and $((\mathfrak{G}, g), X)$ is defined as $((\mathscr{T}, \mu), X) \sqcup_{\mathscr{E}}((\mathfrak{G}, g), X)=((H, h), X)$ where $H=\mathscr{T} \widetilde{\mathfrak{G}}$ and $h=\mu \bar{\Xi}$.

It is given that $\mathscr{T}(\Theta) \supseteq \mathscr{T}(\sigma), \mu(\Theta) \leq \mu(\sigma)$ and $\mathfrak{G}(\Theta) \supseteq \mathfrak{G}(\sigma), g(\Theta) \leq g(\sigma)$ then $\mathscr{T}(\Theta) \cap \mathfrak{G}(\Theta) \supseteq \mathscr{T}(\sigma) \cap \mathfrak{G}(\sigma)$

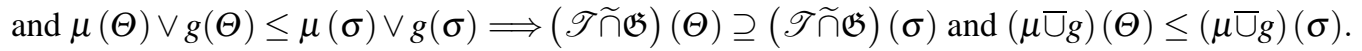


Implies $H(\Theta) \supseteq H(\sigma)$ and $h(\Theta) \leq h(\sigma)$. Further, $\mathscr{T}(\sigma) \supseteq \mathscr{T}(\sigma \star \eta) \cap \mathscr{T}(\eta), \mu(\sigma) \leq \mu(\sigma \star \eta) \vee \mu(\eta)$ and $\mathfrak{G}(\sigma) \supseteq \mathfrak{G}(\sigma \star \eta) \cap \mathfrak{G}(\eta), g(\sigma) \leq g(\sigma \star \eta) \vee g(\eta)$, we $\mathscr{T}(\sigma) \cap \mathfrak{G}(\sigma) \supseteq(\mathscr{T}(\sigma \star \eta) \cap \mathscr{T}(\eta)) \cap(\mathfrak{G}(\sigma \star \eta) \cap \mathfrak{G}(\eta))$ and

$\mu(\sigma) \vee g(\sigma) \leq(\mu(\sigma \star \eta) \vee \mu(\eta)) \vee(g(\sigma \star \eta) \vee g(\eta)), \Longrightarrow \mathscr{T}(\sigma) \cap \mathfrak{G}(\sigma) \supseteq(\mathscr{T}(\sigma \star \eta) \cap \mathfrak{G}(\sigma \star \eta)) \cap(\mathfrak{G}(\eta) \cap \mathfrak{G}(\eta))$ and $\mu(\sigma) \vee g(\sigma) \leq(\mu(\sigma \star \eta) \vee g(\sigma \star \eta)) \vee(g(\eta) \vee g(\eta)), \Longrightarrow(\mathscr{T} \cap \mathfrak{G})(\sigma) \supseteq(\mathscr{T} \widetilde{\mathfrak{G}})(\sigma \star \eta) \cap(\mathscr{T} \cap \mathfrak{G})(\eta)$ and

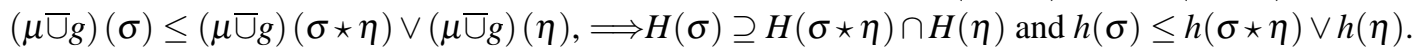

Hence, result is proved.

Note that, intersection of $((\mathscr{T}, \mu), X)$ and $((\mathfrak{G}, g), X)$ over $(U,[0,1])$ is also said to be extended int-uni of $((\mathscr{T}, \mu), X)$ and $((\mathfrak{G}, g), X)$, known as extended int-uni doubt double T-soft fuzzy ideal of $X$.

Theorem 8. Let $\left\{\left(\left(\mathscr{T}_{j}, \mu_{j}\right), X\right)\right.$ is the collection of ideals of $\left.X: j=1,2,3, \ldots\right\}$. Then their Intersection is also a doubt DF-soft fuzzy ideal of BF-algebra $X$.

Definition 17. If $\left(\left(\mathscr{T}_{1}, \mu_{1}\right), X\right)$ and $\left(\left(\mathscr{T}_{2}, \mu_{2}\right), X\right)$ be two doubt DF-soft fuzzy ideal of $X$ over $(U,[0,1])$ the their intersection product is defined as

$$
\left\{\begin{array} { l } 
{ \mathscr { T } _ { 1 } \wedge \mathscr { T } _ { 2 } : X \times X \longrightarrow P ( U ) } \\
{ \mu _ { 1 } \vee \mu _ { 2 } : X \times X \longrightarrow [ 0 , 1 ] }
\end{array} , \quad \text { suchthat } \quad ( \sigma , \eta ) \longrightarrow \left\{\begin{array}{c}
\mathscr{T}(\sigma) \cap \mathscr{T}(\eta) \\
\mu(\sigma) \vee \mu(\eta)
\end{array}\right.\right.
$$

and denoted by $\left(\left(\mathscr{T}_{\wedge}, \mu_{\vee}\right), X ? X\right)$.

Theorem 9. Let $\left(\left(\mathscr{T}_{1}, \mu_{1}\right), X\right)$ and $\left(\left(\mathscr{T}_{2}, \mu_{2}\right), X\right)$ be two doubt DF-soft fuzzy ideal of $X$ over $(U,[0,1])$ then prove that their intersection product is also doubt DF- soft fuzzy ideal of $X \times X$.

Proof. Let for any $(\sigma, \eta),(y, z) \in X \times X$, we have

$$
\begin{aligned}
\left(\mathscr{T}_{1} \wedge \mathscr{T}_{2}\right)(\Theta, \Theta) & =\mathscr{T}_{1}(\Theta) \cap \mathscr{T}_{2}(\Theta) \supseteq \mathscr{T}(\sigma) \cap \mathscr{T}_{(\eta)}=\left(\mathscr{T}_{1} \wedge \mathscr{T}_{2}\right)(\sigma, \eta) \\
& \Longrightarrow\left(\mathscr{T}_{1} \wedge \mathscr{T}_{2}\right)(\Theta, \Theta) \supseteq\left(\mathscr{T}_{1} \wedge \mathscr{T}_{2}\right)(\sigma, \eta)\left(\mu_{1} \vee \mu_{2}\right)(\Theta, \Theta)=\mu_{1}(\Theta) \vee \mu_{2}(\Theta), \leq \mu_{1}(\sigma) \vee \mu_{2}(\eta) \\
& \Longrightarrow\left(\mu_{1} \vee \mu_{2}\right)(\Theta, \Theta) \leq \mu_{1} \vee \mu_{2}(\sigma, \eta)
\end{aligned}
$$

Since $(\sigma, \eta),(y, z) \in X \times X$, we have $\left(\mathscr{T}_{1} \wedge \mathscr{T}_{2}\right)(\sigma, \eta)=\mathscr{T}_{1}(\sigma) \cap \mathscr{T}_{2}(\eta)$. Since, $\left(\left(\mathscr{T}_{1}, \mu_{1}\right), X\right)$ and $\left(\left(\mathscr{T}_{2}, \mu_{2}\right), X\right)$ both are ideals so we have

$$
\begin{aligned}
& \left(\mathscr{T}_{1} \wedge \mathscr{T}_{2}\right)(\sigma, \eta)=\mathscr{T}_{1}(\sigma) \cap \mathscr{T}_{2}(\eta) \supseteq\left(\mathscr{T}_{1}(\sigma \star y) \cap \mathscr{T}_{1}(y)\right) \cap\left(\mathscr{T}_{2}(\eta \star z) \cap \mathscr{T}_{2}(z)\right) \\
& =\left(\mathscr{T}_{1}(\sigma \star y) \cap \mathscr{T}_{2}(\eta \star z)\right) \cap\left(\mathscr{T}_{1}(y) \cap \mathscr{T}_{2}(z)\right)=\left(\left(\mathscr{T}_{1} \wedge \mathscr{T}_{2}\right)(\sigma \star y, \eta \star z)\right) \cap\left(\left(\mathscr{T}_{1} \vee \mathscr{T}_{2}\right)(y, z)\right) \\
& =\left(\left(\mathscr{T}_{1} \vee \mathscr{T}_{2}\right)((\sigma, \eta) \star(y, z))\right) \cap\left(\left(\mathscr{T}_{1} \vee \mathscr{T}_{2}\right)(y, z)\right) \\
& \Longrightarrow\left(\mathscr{T}_{1} \vee \mathscr{T}_{2}\right)(\sigma, \eta) \supseteq\left(\left(\mathscr{T}_{1} \vee \mathscr{T}_{2}\right)((\sigma, \eta) \star(y, z))\right) \cap\left(\left(\mathscr{T}_{1} \vee \mathscr{T}_{2}\right)(y, z)\right)\left(\mu_{1} \vee \mu_{2}\right)(\sigma, \eta)=\mu_{1}(\sigma) \vee \mu_{2}(\eta) \\
& \leq\left(\mu_{1}(\sigma \star y) \vee \mu_{1}(y)\right) \vee\left(\mu_{2}(\eta \star z) \vee \mu_{2}(z)\right)=\left(\mu_{1}(\sigma \star y) \vee \mu_{2}(\eta \star z)\right) \vee\left(\mu_{1}(y) \vee \mu_{2}(z)\right) \\
& =\left(\left(\mu_{1} \vee \mu_{2}\right)(\sigma \star y, \eta \star z)\right) \vee\left(\left(\mu_{1} \vee \mu_{2}\right)(y, z)\right)=\left(\left(\mu_{1} \vee \mu_{2}\right)((\sigma, \eta) \star(y, z))\right) \vee\left(\left(\mu_{1} \vee \mu_{2}\right)(y, z)\right) \\
& \Longrightarrow\left(\mu_{1} \vee \mu_{2}\right)(\sigma, \eta) \leq\left(\left(\mu_{1} \vee \mu_{2}\right)((\sigma, \eta) \star(y, z))\right) \vee\left(\left(\mu_{1} \vee \mu_{2}\right)(y, z)\right)
\end{aligned}
$$

Hence, $\left(\left(\left(\mathscr{T}_{1} \wedge \mathscr{T}_{2}\right),\left(\mu_{1} \vee \mu_{2}\right)\right), X \times X\right)$ is a "DDF-soft fuzzy algebra" over $(U,[0,1])$.

\section{Conclusion}

We discussed the DF-soft fuzzy BF-algebra, doubt DF-soft fuzzy BF-algebra and their ideals. By using union product and intersection product, the above mentioned concepts are investigated. We can easily see that DF-soft algebra is not also a double framed B-soft algebra by 1.Example. Similar case for DFSB-soft fuzzy ideal and DFT-soft fuzzy ideal. 


\section{Competing interests}

The authors declare that they have no competing interests.

\section{Authors' contributions}

All authors have contributed to all parts of the article. All authors read and approved the final manuscript.

\section{References}

[1] Phuong, N. H., \& Kreinovich, V. 2001. Fuzzy logic and its applications in medicine. International journal of medical informatics,62(2-3), 165-173.

[2] Zadeh, L. A. 1996. Fuzzy sets. In Fuzzy Sets, Fuzzy Logic, And Fuzzy Systems: Selected Papers by Lotfi A Zadeh, (pp. 394-432).

[3] Molodtsov, D. 1999. Soft set theory-first results. Computers \& Mathematics with Applications, 37(4-5), 19-31.

[4] Maji, P. K., Roy, A. R., \& Biswas, R. 2002. An application of soft sets in a decision making problem. Computers \& Mathematics with Applications, 44(8-9), 1077-1083.

[5] Maji, P. K., Biswas, R., \& Roy, A. 2003. Soft set theory. Computers \& Mathematics with Applications, 45(4-5), 555-562.

[6] Aktaş, H., \& Çă̆man, N. 2007. Soft sets and soft groups. Information sciences, 177(13), 2726-2735.

[7] Ali, M. I., Feng, F., Liu, X., Min, W. K., \& Shabir, M. 2009. On some new operations in soft set theory. Computers \& Mathematics with Applications, 57(9), 1547-1553.

[8] Jun, Y. B., \& Ahn, S. S. 2012. Double-framed soft sets with applications in BCK/BCI-algebras. Journal of Applied Mathematics, 2012 .

[9] Cho, Y. U., Lee, K. J., \& Jun, Y. B. 2015. A study on double-framed soft near-rings. Applied Mathematical Sciences, 9(18), 867-873

[10] Naz. M. 2015. Some studies on algebraic structure of soft sets. P.hd Dissertion Quaid-i-Azam University Islamabad, Pakistan .

[11] Hadipour, A. R. 2014. Double-framed Soft BF-algebras. Indian Journal of Science and Technology, 7(4), 491-496.

[12] Shabir, M. \& Khan, S. 2016. Double framed soft topological space, Annals of Fuzzy Mathematics and Informations, x(x), 1-xx.

[13] Khan, M. B., \& Mahmood, T. 2018. Applications of Double Framed T-Soft Fuzzy Sets in BCK/BCI-Algebras.

[14] Barbhuiya, S. R. Doubt fuzzy ideals of BF-algebra. IOSR Journal of Mathematics (IOSR-JM), 10(2), 65-70.

[15] Walendziak, A. 2007. On BF-algebras. Mathematica Slovaca, 57(2), 119-128.

[16] Mahmood, T., Ullah, M., Khan, M. B., \& Ullah, K. 2017. On Twisted Soft Ideal of Soft Ordered Semigroups. Journal of Information Communication Technologies and Robotics Applications (JICTRA).(Formally known as Journal of Computer Science of NICE). ISSN\# 2226-3683, 8, 74-85.

[17] Acar, U., Koyuncu, F., \& Tanay, B. 2010. Soft sets and soft rings. Computers \& Mathematics with Applications, 58(11), 3458-3463.

[18] Sezer, A. S., \& Atagün, A. O. 2016. A New Kind of Vector Space: Soft Vector Space. Southeast Asian Bulletin of Mathematics, 40(5), 753-770.

[19] Ali, M. I., Shabir, M., \& Feng, F. 2017. Representation of graphs based on neighborhoods and soft sets. International Journal of Machine Learning and Cybernetics, 8(5), 1525-1535.

[20] Jun, Y. B., Kim, H. S., \& Neggers, J. 2009. Pseudo d-algebras. Information Sciences, 179(11), 1751-1759.

[21] Jun, Y. B., Lee, K. J., \& Khan, A. 2010. Soft ordered semigroups. Mathematical Logic Quarterly, 56(1), 42-50.

[22] Jun, Y. B., Lee, K. J., \& Park, C. H. 2009. Soft set theory applied to ideals in d-algebras. Computers \& Mathematics with Applications, 57(3), 367-378.

[23] Jun, Y. B., Lee, K. J., \& Zhan, J. 2009. Soft p-ideals of soft BCI-algebras. Computers \& Mathematics with Applications, 58(10), 2060-2068.

[24] Hayat, K., Cao, B. Y., Mahmood, T., \& Tariq, K. U. H. 2016. Applications of double-framed soft ideals in BE-algebras. New trends in Mathematical Sciences, 4(1), 285-295.

[25] Khan, M. B, Çetkin, V., Mahmood, T., Jan, N., Ullah, K., Aygu, H. 2019. Near rings in the view of double-framed soft fuzzy sets. Sigma journal of engineering and natural sciences 37(1), 175-186. 